\title{
Travelling convection vortices in the ionosphere map to the central plasma sheet
}

\author{
A. Yahnin*, T. Moretto \\ Danish Meteorological Institute, Solar-Terrestrial Physics Division, Copenhagen, Denmark
}

Received: 29 November 1995/Revised: 22 April 1996/Accepted: 21 May 1996

\begin{abstract}
We investigate the magnetospheric domain responsible for the generation of ionospheric travelling convection vortices (TCV) by comparing the location of the TCV to the locations of the low-altitude particle-precipitation boundaries deduced from the DMSP satellite measurements. For three very well documented TCV events we are able to identify suitable satellite passes, in the sense that for each event we can identify two to three passes occurring close to the TCV observation in both time and space. In all three cases the comparisons place the TCV centres at or equatorward of the central plasma sheet/boundary plasma sheet precipitation boundary. Thus our results indicate that the field-aligned currents related to the TCV originate in the plasma sheet rather than at the magnetopause or in the low-latitude boundary layer, as previous studies suggest.
\end{abstract}

\section{Introduction}

The question of generation mechanisms for the travelling convection vortices (TCV) signatures which are closely related to the magnetic impulsive events observed, most often, in the pre-noon high-latitude ionosphere, is still largely unsolved. All theoretical studies, so far, have considered sources at the magnetopause or in the low-latitude boundary layer (LLBL), (e.g. Glassmeier, 1992; Lysak et al., 1994, and references therein). On the basis of multiinstrumental observations many authors (Heikkila et al., 1989; Mende et al., 1990; Potemra et al., 1992; Vorobjev, 1993; Lühr et al., 1996) agree on the conclusion that magnetic impulsive events and TCVs occur on closed field lines. Most of them also suggest, nevertheless, that these

\footnotetext{
* Permanent address: Polar Geophysical Institute, Apatity, Murmansk region, 184200, Russia (E-mail: yahnin@pgi-ksc.murmansk.su) Correspondence to: A. Yahnin
}

closed field lines map to the LLBL. The only direct experimental verification of this, as yet, is provided by McHenry et al. (1990), who examined the location of the vortices related to continuous geomagnetic pulsation events relative to the particle-precipitation boundaries obtained from the measurements made on board the DMSP satellites, and concluded that the vortices are located close to the equatorward edge of the LLBL. This led the authors to suggest the Kelvin-Helmholtz instability on the LLBL inner edge as the source of the chain of field-aligned currents producing the vortices of the Hall current in the ionosphere. However, it is not obvious that this conclusion applies to the vortices related to magnetic impulsive events, which are generally believed to be of a different nature. The recent result for a case study reported by Yahnin et al. (1996) suggests that the locations of the vortices map to inside the plasma sheet rather than to the LLBL.

In this report we carry out a similar analysis of the relations between TCVs and DMSP particle boundaries for three events carefully chosen to be well documented and often cited in the literature as well-defined impulsive TCV events. The first event is the very first, defining TCV event described by Friis-Christensen et al. (1988) in their pioneering paper. Next is the well-known event studied by Vogelsang et al. (1993), unique in that the field-aligned currents of the TCV are inferred using the VIKING satellite measurements. Finally, for the third event we shall present that of Lühr et al. (1996). This is the most recently published TCV event and it is analysed on the basis of the most comprehensive ground-based data set including the data from widespread magnetometer networks, all-sky camera and photometer data, and data on the ionospheric electric field from the EISCAT radar measurements.

Naturally, it is very difficult to find DMSP passes coinciding in space and time with such short-lived and local phenomena as TCVs. We do not have perfect coincidence for any of the events presented here, but for each of them we have been able to identify several passes quite close to the location of the event (or its magnetically 
conjugated location) at times shortly before and after the event. Combined with additional observational evidence of the location of the particle precipitation boundaries, this allows us to confine quite reliably the locations of the boundaries during each of the events. The various precipitation domains are identified in this study using the automatic, neural-network routine of Newell et al. (1991).

As discussed by Lühr et al. (1996), evidence exists that the ground magnetic field variations of the TCV signatures are indeed produced by Hall-current vortices which, in turn, must be driven by field-aligned current filaments in their centre. For all three events our analyses agree strikingly well on the result that the locations of the TCV centres map to regions in the central plasma sheet (CPS). This leads us to consider this result a significant challenge to the common view of the LLBL as the source region for TCVs, in spite of the obvious limitations of this study given by the small number of events, the lack of exact satellite overpasses and the use of the automatic routine for determination of the particle boundaries.

In the next section we present the details of our analysis for each of the events. Then follows a discussion of our approach and our results, and in the final section we give a summary.

\section{Data presentation}

For each event we summarise our findings in two figures displaying the locations of the TCV and the precipitation boundaries on maps in geographic coordinates and Inv. Lat.-MLT coordinates, respectively. The map in geographic coordinates serves to show the positions of the satellite trajectories relative to the stations where the TCV signatures are observed. Because the universal times of the TCV observations often differ from the times of the satellite passes, the second type of map is needed to illustrate how well the satellite tracks and the TCV positions match in Invariant Latitude and Magnetic Local Time.

\subsection{Event 1}

This famous event of Friis-Christensen et al. (1988), appearing on the cover of the Geophysical Research Letters, was observed over western Greenland between 1005 and 1020 UT on 28 June 1986 . As is evident from the analysis of the rotation of the convection vectors [see Fig. 4 of Friis-Christensen et al. (1988)], the centres of the twinconvection vortex system pass just overhead the stations SKT and DYB at 1013-1014 UT. The vortex system travels from east to west and the locations of the leading vortex over these stations are marked (large circles) in Fig. 1a. The stations of the Greenland west coast were at this time at $\mathrm{MLT}=08$. Three relevant DMSP satellite passes are available for this event. Two of them cross the high-latitude regions of the southern hemisphere conjugate to the Greenland region. Right at the time of the TCV observation one of these, DMSP-F6, passes a region very nearly conjugate to the TCV location. Another conjugated pass occurs about 25 min earlier than the TCV

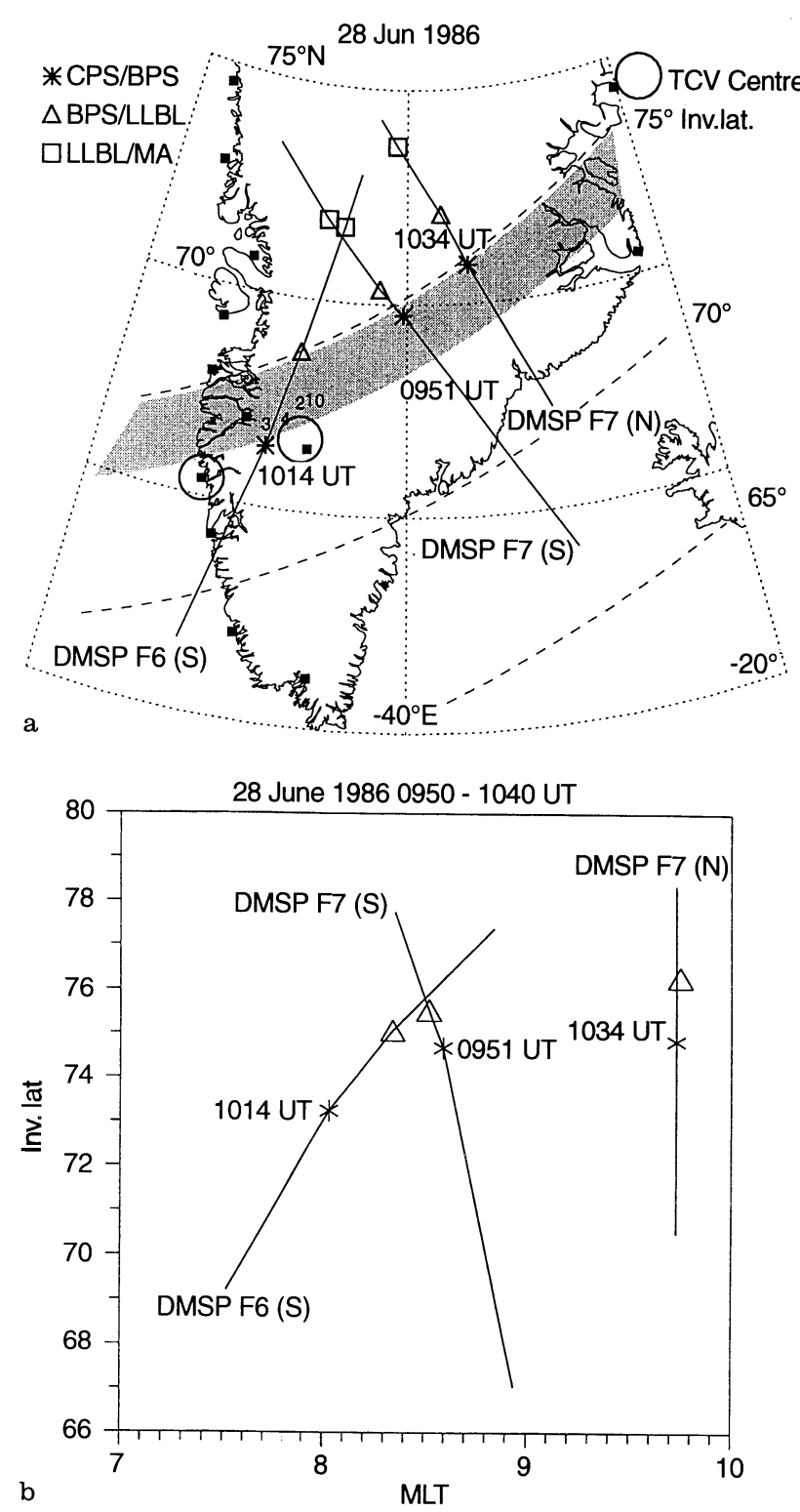

Fig. 1. a Geographic map of the magnetic stations (small filled squares) and the locations of the TCV centres for the event of Friis-Christensen et al. (1988). In addition, the DMSP satellite trajectories are displayed and on these the detected precipitation boundaries are marked according to the legend in the figure. For further details see the text. b The satellite trajectories of a, including the locations of the precipitation boundaries, are displayed in the Inv. Lat.-MLT coordinate system

observation. This passes over the TCV latitudes at MLT $=8.7$. The third pass occurs over the northern hemisphere some 20 min after the event at MLT $=9.7$. The trace of each of these satellite passes is displayed in Fig. 1a, b in the two different coordinate systems, and on each trace the precipitation boundaries detected during that pass are marked. The most equatorward mark is that of the boundary between the precipitation signatures corresponding to the CPS and the boundary plasma sheet (BPS). The time displayed by each trace is the UT of passage of this boundary. The next mark poleward is of the BPS/LLBL boundary, and the last mark indicates the 
boundary between LLBL and Mantle precipitation. For the southern passes we shall note that the conjugation is calculated using the international geomagnetic reference field. The conjugation of points is, in fact, influenced by the external field, and to estimate the effect of this on our results, we also calculate the projection to the northern hemisphere of the location of the CPS/BPS boundary measured by the DMSP-F6 using the magnetospheric magnetic-field model of Tsyganenko (1989). This model allows for various external field contributions parameterised by the $K_{P}$ index. The positions of the conjugated points resulting from values of $K_{P}$ of $0-4$ are indicated in Fig. 1a by the small numbers around the CPS/BPS boundary mark (For $K_{P}$ of 5 and 6 in the model the satellite locations are on open field lines). It is evident that for a wide range of external field configurations, the projection of the CPS/BPS boundary is not shifted equatorward further than the latitude of the marked conjugated point. Thus, we feel safe in concluding that the DMSP-F6 satellite meets the TCV centre just equatorward of the CPS/BPS boundary. The measurements made during the northern and southern passes of the DMSP-F7 satellite further confirm this result. Both of these passes occur eastward of the magnetometer chain where the TCV is observed. We expect the TCV to have been in this region a few minutes earlier than the time at which it is observed at the west coast at approximately the same invariant latitude. We observe that both before and after the TCV event the CPS/BPS boundary is detected at almost exactly the same invariant latitude well poleward of the presumed TCV trajectory. On this basis alone we cannot, of course, completely exclude the possibility that the configuration of the boundaries could undergo some changes during the 40-min time-interval between these passes. Unfortunately, no additional observations (e.g. optical as for Event 3) to confirm the location and/or stability of the boundaries are available for this event. In Fig. 1a we have shaded the region confined by the most equatorward latitudes of the CPS/BPS and BPS/LLBL boundaries, respectively, determined for the three satellite passes. It is observed that the TCV centres, and their trajectory, lie slightly equatorward of this region and consequently as far as several degrees equatorward of the BPS/LLBL boundary. As already argued we conclude from this that the source region of the TCV event is in the CPS.

\subsection{Event 2}

A unique study of the field-aligned currents related to the TCV event occurring on 21 April 1986 around 0610-0620 UT over Scandinavia was made by Vogelsang et al. (1983). They used the data from the VIKING satellite; at the time of the event, this is at an altitude of roughly $2 R_{E}$, and its trajectory maps along magnetic field lines to the region near Svalbard. Figure 2a, b displays the VIKING ionospheric footprints [after Fig. 1 of Vogelsang et al. (1993)] along with the traces of the DMSP-F7 satellite passes over these latitudes some $15 \mathrm{~min}$ before the event in the southern hemisphere and $20 \mathrm{~min}$ after the event in the northern hemisphere at MLT $=8.6-9.0$ and
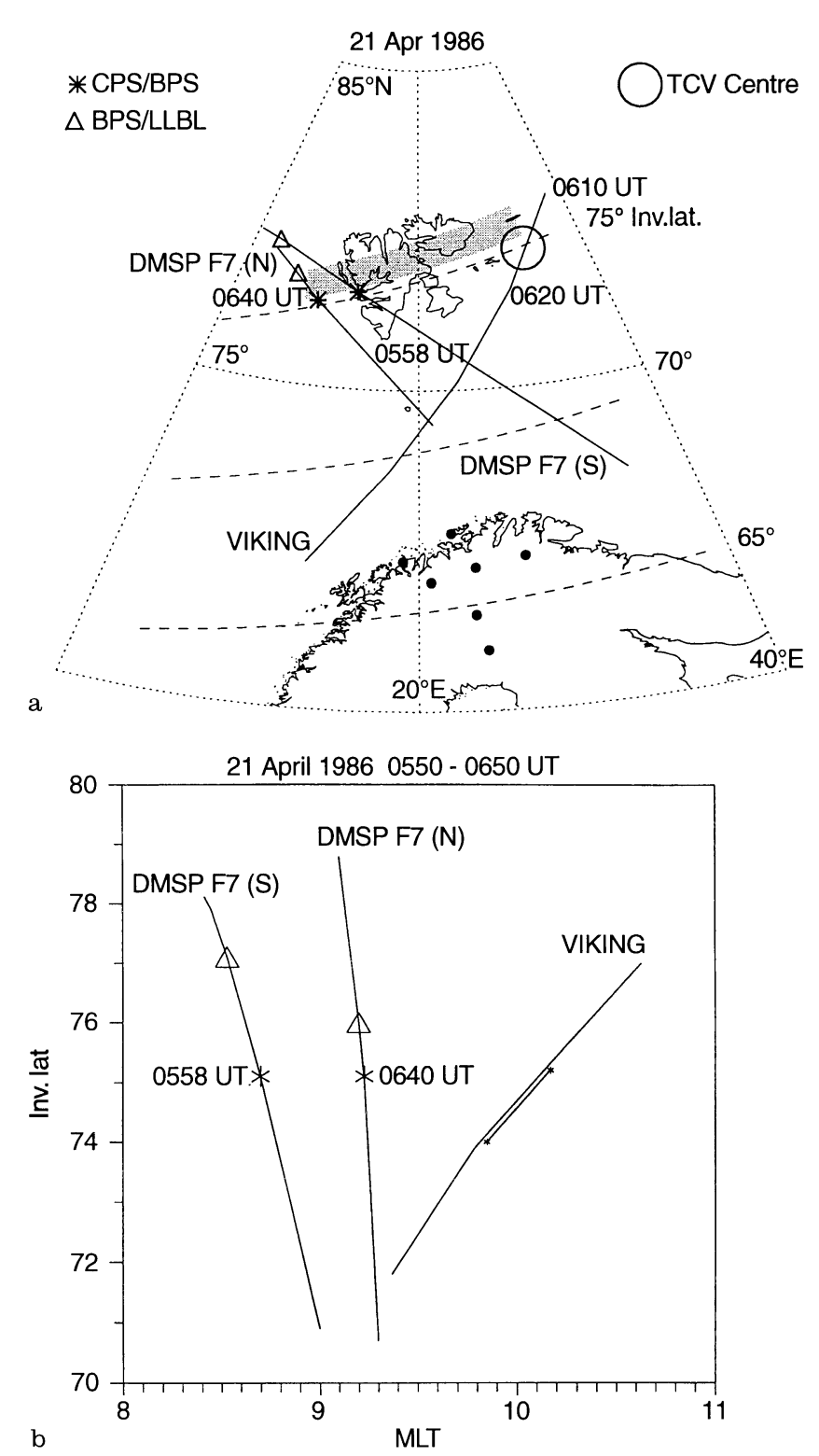

Fig. 2. a Geographic map of the magnetic stations (small filled circles) and the location of the TCV centres for the event of Vogelsang et al. (1993). In addition, the VIKING and the DMSP satellite trajectories are displayed and on the latter the detected precipitation boundaries are marked according to the legend in the figure. For further details see the text. b The aforementioned satellite trajectories including the locations of the precipitation boundaries, are displayed in the Inv. Lat.-MLT coordinate system

MLT $=9.2-9.3$, respectively. According to the description of the event made by Vogelsang et al. (1993) and by Lühr and Blawert (1994), the VIKING satellite encounters the $\mathrm{TCV}$ at an invariant latitude in the range $74^{\circ}-75^{\circ}$ at $\mathrm{MLT}=10$. This position is marked by a large circle in Fig. 2a and by a line in Fig. 2b. We mark the CPS/BPS and BPS/LLBL boundaries as determined by the satellite on the DMSP traces in the same way as for the previous event. While the BPS/LLBL boundary shifts between passes by one degree of invariant latitude (cf. Fig. 2b), the CPS/BPS boundary is observed at the same invariant latitude of $75.10^{\circ}$. Consequently, we conclude that the 
TCV trajectory is observed just equatorward of the CPS/BPS boundary, which means that the TCV source region is in the CPS. An additional argument for this is provided in the original paper (Vogelsang et al., 1993). There it is noted that ". . . the particle detectors on VIKING first observed energetic $(>10 \mathrm{keV})$ electrons and ions at 0608 UT, heralding the encounter with the ring-current population and indicating that VIKING had left the cusp and reached the closed-field-line region". We should like to add that before the TCV event the satellite moves further southward for several minutes and that the presence of the energetic ions means that the satellite has entered the plasma-sheet population, and so we interpret this observation as evidence that VIKING meets the TCV well inside the magnetosphere.

\subsection{Event 3}

A very detailed study of a TCV event on 6 January 1992 is presented by Lühr et al. (1996). The map of stations used for this study is shown in Fig. 3a. The locations of the TCV centres, which are observed at around 0755 UT over Scandinavia and some minutes later at the east coast of Greenland, are obtained from the analysis made by Lühr et al. (1996) and are displayed on the map. The F9 satellite passes the regions of interest in the northern and southern hemispheres $35 \mathrm{~min}$ before and $20 \mathrm{~min}$ after the event, respectively, and the F11 satellite passes through this region $30 \mathrm{~min}$ after the event. The trajectories of the satellites in the two different sets of coordinates are displayed in Fig. 3a, b. From the latter it is evident that the southern pass of the F9 satellite is at MLT $=08$, the northern pass of this satellite is at MLT $=09$, and the F11 satellite is at MLT $=08-10$ during its traverse of the region. The TCV is registered at $\mathrm{MLT}=10$ in the Scandinavian sector and at $\mathrm{MLT}=08$ over Greenland. No LLBL signature was registered by the DMSP satellite at all during this event, so only the CPS/BPS and BPS/Mantle boundaries are marked on the traces. The boundaries obtained over the time-interval of $1 \mathrm{~h}$ do not exhibit any significant shifts. This agrees well with the optical observations as described in Lühr et al. (1996). Using scanning photometers they observe a band of red luminosity over the northern part of Svalbard and they interpret this as an indication of Mantle-type precipitation. Their Fig. 6 illustrates how the latitudinal position of the red auroral band varies as a function of time. During the interval of 0720-0830 UT, the position of the luminosity band is very stable. Only some minor, not more than $0.5^{\circ}$, short-lasting shifts of the equatorial edge of the band related to the occurrences of the discrete auroras are observed. These observations strongly support our presumption that the boundaries measured by the satellites before and after the event provide a reliable estimate of the locations of the boundaries also during the event. As for the previous events, in Fig. 3a we shade the region between the lowest latitude of the CPS/BPS boundaries and the lowest latitude of the BPS/Mantle boundaries. From the comparison, we observe, once more, that the trajectory of the TCV centres lies equatorward of this region, that is in the CPS region,
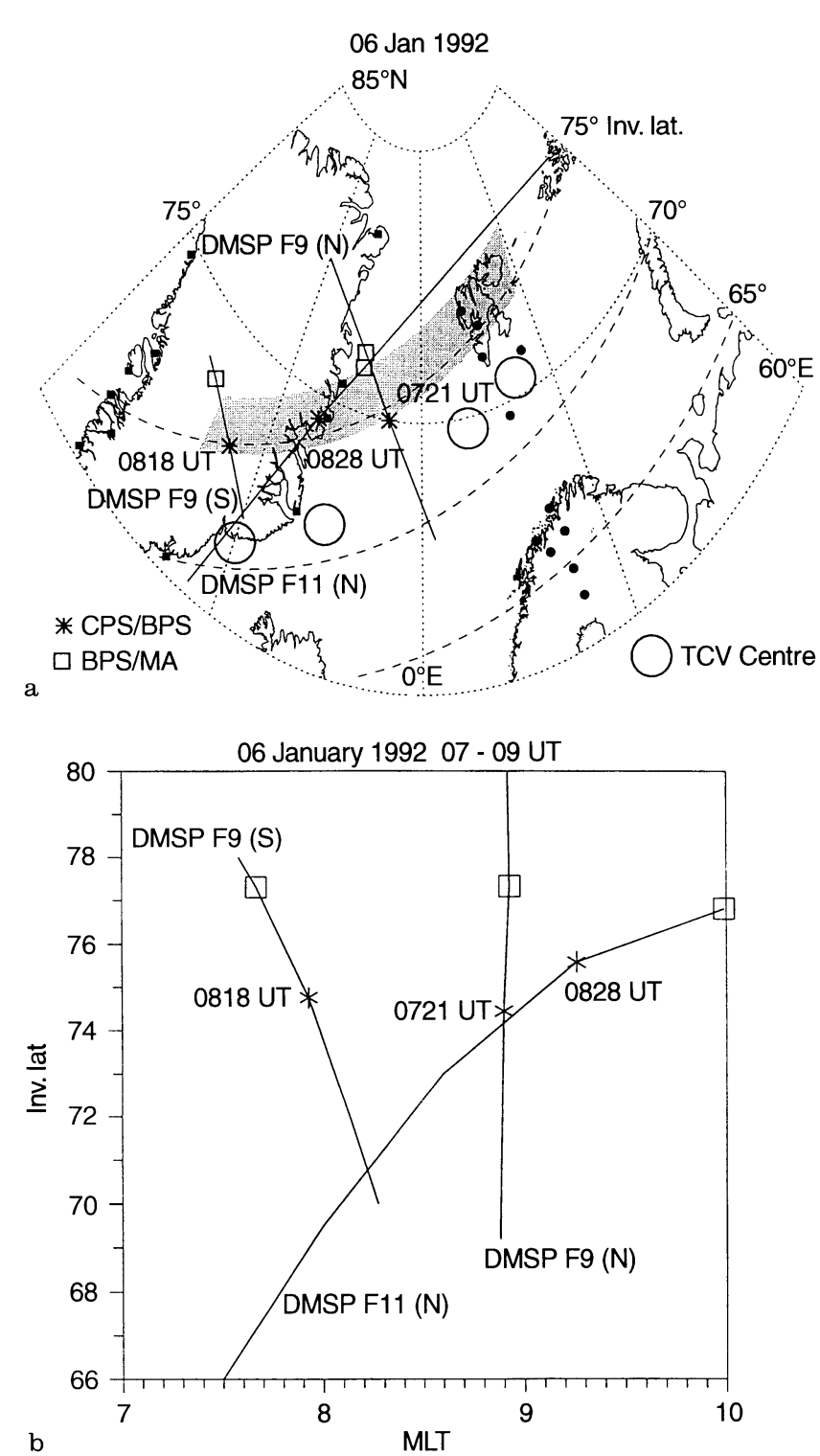

Fig. 3. a Geographic map of the magnetic stations (small filled circles and squares) and the locations of the TCV centres for the event of Lühr et al. (1996). In addition, the DMSP satellite trajectories are displayed and on these the detected precipitation boundaries are marked according to the legend in the figure. For further details see the text. b The aforementioned satellite trajectories including the locations of the precipitation boundaries, are displayed in the Inv. Lat.-MLT coordinate system

and hence far equatorward of the boundary which could be interpreted as the boundary between the open- and closed-field-line regions.

\section{Discussion}

Due to the fact that our result contravenes the prevailing conception that the likely source region for the ionospheric TCV signatures is in the LLBL, we should like to make a special effort to justify our confidence in the result. In the following we first comment on the two most critical points of our analysis and subsequently we relate our result to the few previous publications on this topic. 
First, our conclusion relies on the determination of the boundaries of the particle precipitation. In this study we use the automatic procedure of Newell et al. (1991). This has recently been evaluated in several papers (e.g. de la Beaujardiere et al., 1993; Burke et al., 1993; Yahnin et al., 1996), all of which deal with pre-noon precipitation. Generally, very good agreement is found for the CPS/BPS boundary between the results of the automatic procedure and the results of visually analysing the particle spectrograms. However, a problem was encountered for the determination of the BPS/Mantle boundary when comparing with other observations (Burke et al., 1993) which suggested that the BPS region extends further poleward than is determined by the automatic routine. We should like to note that if this is the case, it would only further strengthen our conclusion that the source region of the TCVs is not in the LLBL or Mantle. An analysis with the same objective as the study presented here of a TCV event in relation to DMSP satellite measurements and optical data was carried out by Yahnin et al. (1996). In this study the CPS/BPS boundary was determined both automatically and visually from the DMSP spectrograms and good agreement was found. Furthermore, the boundary was found to be well co-located with the optically defined boundary between the regions of "green" and "red" luminosity, respectively. It is generally believed that the "green" luminosity (for which the 557.7-nm-emission intensity predominates the intensity of the $630.0-\mathrm{nm}$ emission) is produced by the rather hard electron precipitation which is typical of CPS precipitation. Further support of the reliability of our conclusions is provided by the fact that the automatic-identification scheme provides not only the position of the boundaries but also some characteristics of the precipitation of the various regions (Newell et al., 1991). In particular, the average energies of the particles are supplied, and for all of the events of this study, the precipitated electrons of the region determined as CPS have average energies of several $\mathrm{keV}$, which confirms this interpretation.

It should be noted that the question of the mapping of the precipitation domains is still subject to discussion. For example, Nishida et al. (1993), on the basis of the particle and electric-field measurements from the AKEBONO satellite, conclude that the precipitation of protons in the morning region can be divided into two parts. The equatorial part of the precipitation coinciding with the region of energetic electrons is on closed field lines which map to the plasma sheet, whereas the poleward part of the proton precipitation is on open field lines mapping to the tail lobe. The terms CPS, BPS and others are avoided by Nishida et al. (1993), but in our opinion, the poleward part of the proton precipitation, as defined above, corresponds well to the BPS, LLBL and MA domains, or a mixture thereof, in the scheme of Newell et al. (1991). It is beyond the scope of this report to go any deeper into this discussion. Therefore we shall conclude this point by noting that these differences in the interpretations of the proton-precipitation sources do not affect our results because, in any case, the TCVs map to the region of energetic electrons, which is inside the plasma sheet.
The second aspect of our study on which we wish to comment here, is the lack of satellite passes in closer coincidence in both space and time with the TCV observations. Both Mende et al. (1990) and Potemra et al. (1992) have shown that sometimes the magnetospheric boundaries can shift significantly during magnetic impulsive events and TCV events. In the former work, the dynamics of the red aurora during magnetic impulsive events is considered using all-sky camera observations made at the South Pole. They show that just at the time of the magnetic impulse event the equatorial boundary moves equatorward several degrees, after which it recovers to the pre-event position. On the basis of the VIKING satellite measurements, Potemra et al. (1992) estimate the equatorial shift of the cusp boundary during a TCV event to be as large as $3^{\circ}$. Comparing scanning photometer and magnetometer observations, Vorobjev (1993) also observes that the equatorial border of the red auroral band shifts equatorward during the TCV event but not beyond the location of the TCV centre.

In each of our cases we are able to argue on the basis of the indirect evidence provided by additional observations, that the possible motion of the boundaries does not significantly affect our conclusions. For Event 2 our conclusion is supported by the particle measurements of the VIKING satellite, and for Event 3 by the stability of the luminosity boundary. For the case of Event 1 we have almost perfect coincidence - although in the conjugate hemisphere - between the DMSP overflight and the TCV observation, and we observe that the boundaries as determined from this pass appear somewhat equatorward as compared to the boundaries determined by the two other passes before and after the TCV event, respectively. On the other hand, the TCV centres are observed clearly equatorward even of this most equatorward position of the CPS/BPS boundary, and hence we take this case to show that even if such dynamics of the boundaries exist, as it is suggested above, they are not substantial enough to alter our conclusions. This is further confirmed in Yahnin et al. (1996), where they have almost exact coincidence between the DMSP satellite pass and the TCV observation and reach the same conclusion as this study.

Attempts to determine the magnetospheric sources of TCVs on the basis of ground observations only are presented in the works of Vorobjev (1993) and Lühr et al. (1996). In both papers the TCV centres are observed equatorward of the "red" auroral band which is usually associated with the precipitation from some boundary region, as for example the BPS, LLBL, Mantle or some combination of these. This leads them to conclude that the TCVs are generated well inside the magnetosphere on closed field lines. The same conclusion is expressed in the quote from Vogelsang et al. (1993) included in Sect. 2.2. In their comprehensive multi-instrumental study of a TCV event, Heikkila et al. (1989) consider the location of the TCV relative to the cleft precipitation and the convection reversal boundary by use of scanning photometer data and satellite measurements. They also conclude that the TCV event occurs on closed field lines. The mapping of the ionospheric location of TCV events to the magnetosphere by use of the Tsyganenko (1989) magnetic-field 
model was carried out in the studies of Yahnin et al. (1995) and Lühr et al. (1996) with very similar results (cf. Fig. 8 of the former and Fig. 10 of the latter). The TCV trajectories are found to lie inside the nominal location of the magnetopause at distances as far as $2 R_{E}$ near noon and 5-7 $R_{E}$ at dawn. Both groups of authors suggest, on the basis of this, the inner edge of the LLBL as a guideline for the TCV source. We believe however, that the result of these mappings may just as well be interpreted to mark some inner boundary in the plasma sheet as, for example, the CPS/BPS or the outer edge of the radiation belt. Indeed, noting that Eastman and Hones (1979) report an average thickness of the LLBL of as little as $0.4 R_{E}$, it seems unlikely, even considering the uncertainties of the mapping procedure, repetitively to observe the LLBL as far as $5 R_{E}$ from the magnetopause. This means that there is no real disagreement between these earlier results and ours.

We should like to end this discussion with a few remarks regarding some other magnetic impulsive events presented by Lin et al. (1995). These authors suggest that the TCV signatures are only one of many possible manifestations related to the large number of different types of magnetic impulsive events. In particular, they present examples of travelling impulsive disturbances for which no clear vortex structures can be identified [see also Bering et al. (1990), where two further examples are presented]. The objective of their study was to examine the external conditions during the events in order to obtain indications of the responsible generation mechanisms. They conclude that some, but not all, of the events are likely triggered by pressure pulses in the solar wind, and that some of them are possibly triggered by the FTE (flux transfer event) process. For two of the events we have been able to identify suitable DMSP passes allowing us to examine the relationship of these events to the precipitation boundaries in the same way as for the TCV events. The two events occur on 14 January 1986 around 1130 UT [c.f. Fig. 5 of Bering et al. (1990)] and 1255 UT [c.f. Fig. 4 of Lin et al. (1995)]. The first event belongs to the group of "possible-FTE" events and the second to the group of "likely pressure-pulse" events, as defined in Lin et al. (1995). For these events we have chosen not to use the vortex centres to determine the source region because the possible vortex structures are far less obvious than for the previous cases. Instead, we take the latitude where the magnetic disturbances are largest as a signature of the location of the source of the current system. For both events the comparison with the DMSP boundaries clearly places this location equatorward of the CPS/BPS boundary, and hence we reach the same conclusion as for the TCV events, that the source region of the field-aligned currents that drive the events is in the CPS. We should like to note that this makes it hard to believe the FTE process, being a process at the magnetopause, to be directly responsible for the generation of the field-aligned currents of one of these events.

\section{Summary}

In conclusion, a re-examination of three typical TCV events including the determinations of particle boundaries obtained from low-altitude satellite measurements shows that TCVs map to regions inside the plasma sheet which are well separated from the LLBL/Mantle region by BPStype precipitation.

Acknowledgements. We gratefully appreciate the on-line DMSP database facility at APL (Newell et al., 1991) from which this study has benefited greatly. We wish to thank E. Friis-Christensen for his encouragement and useful discussions. A. Y. would like to thank the Danish Meteorological Institute, where this work was done, for its hospitality during his stay there and the Nordic Baltic Scholarship Scheme for its financial support of this stay.

Topical Editor K.-H. Glassmeier thanks M. J. Engebretson and $\mathrm{H}$. Lühr for their help in evaluating this paper.

\section{References}

Beaujardiere, O. de la, J. Watermann, P. Newell, and F. Rich, Relationship between Birkeland current regions, particle precipitation, and electric field, J. Geophys. Res., 98, 7711-7720, 1993.

Bering, E. A., L. J. Lanzerotti, J. R. Benbrook, C. G. Maclennan, A. Wolfe, R. E. Lopez et al., Solar wind properties observed during high-latitude impulsive perturbation events, Geophys. Res. Lett. 17, 579-582, 1990.

Burke, W. J., B. Jacobsen, P. E. Sandholt, W. F. Dening, N. C. Maynard, and P. T. Newell, Optical signatures and sources of prenoon auroral precipitation, J. Geophys. Res., 98, $11521-11529,1993$.

Eastman, T. E., and E. W. Hones Jr., Characteristics of the boundary layer and magnetopause layer as observed by IMP-6, J. Geophys. Res., 84, 2019-2028, 1979.

Friis-Christensen, E., M. A. McHenry, C. R. Clauer, and S. Vennerstrøm, Ionospheric travelling convection vortices observed near the polar cleft: a triggered response to sudden changes in the solar wind, Geophys. Res. Lett., 15, 253-256, 1988.

Glassmeier, K-H., Travelling magnetospheric convection twin-vortices: observations and theory, Ann. Geophysicae, 10, 547-565, 1992.

Heikkila, W. J., T. S. Jørgensen, L. J. Lanzerotti, and C. G. Maclennan, A transient auroral event on the dayside, J. Geophys. Res., 94, 15 291-15 305, 1989.

Lin, Z. M., E. A. Bering, J. R. Benbrook, B. Liao, L. J. Lanzerotti, C. G. Maclennan et al., Statistical studies of impulsive events at high latitudes, J. Geophys. Res., 100, 7553-7556, 1995.

Lühr, H., and W. Blawert, Ground signatures of travelling convection vortices, in Solar Wind Sources of Magnetospheric ULF Waves, Eds. M. J. Engebretson, K. Takanashi, and M. Scholer, Vol. 81 of Geophys. Monogr., pp. 231-251, AGU, 1994.

Lühr, H., M. Lockwood, P. E. Sandholt, T. L. Hansen, and T. Moretto, Multi-instrument ground-based observations of a travelling convection vortices event, Ann. Geophysicae, 14, 162-181, 1996.

Lysak, R. L., Y. Song, and D. H. Lee, Generation of the ULF waves by fluctuations in the magnetopause position, in Solar Wind Sources of Magnetospheric ULF Waves, Eds. M. J. Engebretson, K. Takanashi, and M. Scholer, Vol. 81 of Geophys. Monogr., pp. 273-281, AGU, 1994.

McHenry, M. A., C. R. Clauer, E. Friis-Christensen, P. T. Newell, and J. D. Kelly, Ground observations of magnetospheric boundary layer phenomena, J. Geophys. Res., 95, 14995-15005, 1990.

Mende, S. B., R. L. Rairden, L. J. Lanzerotti, and C. G. Maclennan, Magnetic impulses and associated optical signatures in the dayside aurora, Geophys. Res. Lett., 17, 131-134, 1990.

Newell, P. T., S. Wing, C.-I. Meng, and S. Sigillito, The auroral oval position, structure and intensity of precipitation from 1984 onwards: an automated on-line database, J. Geophys. Res., 96, 5877-5882, 1991. 
Nishida, A., T. Mukai, H. Hayakawa, A. Matsuoka, K. Tsuruda, N. Kaya, and H. Fukunishi, Unexpected features of the ion precipitation in the so-called cleft/low-latitude boundary layer region: association with sunward convection and occurrence on open field lines, J. Geophys. Res., 98, 11 161-11 176, 1993.

Potemra, T. A., R. E. Erlandson, L. J. Zanetti, R. L. Arnoldy, J. Woch, and E. Friis-Christensen, The dynamic cusp, J. Geophys. Res., 97, 2835-2844, 1992.

Tsyganenko, N. A., A magnetospheric magnetic field model with a warped tail current sheet, Planet. Space Sci., 37, 5-20, 1989.

Vogelsang, H., H. Lühr, H. Voelker, J. Woch, T. Bösinger, T. A. Potemra, and P.-A. Lindqvist, An ionospheric travelling convec- tion vortex event observed by ground-based magnetometers and by VIKING, Geophys. Res. Lett., 20, 2343-2346, 1993.

Vorobjev, V. G., Dynamics of the Hall current vortices on the dayside high latitudes, Geomagn. and Aeron., 33, 69-79, In Russian (English translation: Vol. 33, 612-620, 1994), 1993.

Yahnin, A. G., E. Titova, A. Lubchich, T. Bösinger, J. Manninin, T. Turunen, T. Hansen et al., Dayside high-latitude magnetic impulsive events: their characteristics and relationship to the sudden impulses, J. Atmos. Terr. Phys., 57, 1569-1582, 1995.

Yahnin, A. G., V. G. Vorobjev, T. Bösinger, R. Rasinkangas, D. G. Sibeck, and P. T. Newell, On the source region of the travelling convection vortices, Geophys. Res. Lett., accepted, 1996. 